\title{
Influence of Buried Hydrogen-Bonding Groups within Monolayer Films on Gas-Surface Energy Exchange and Accommodation
}

\author{
M. K. Ferguson, J. R. Lohr, B. S. Day, and J. R. Morris \\ Department of Chemistry, Virginia Tech, Blacksburg, Virginia 24061, USA
}

(Received 23 September 2003; published 19 February 2004)

\begin{abstract}
Self-assembled monolayers (SAMs) of carbonyl-containing alkanethiols on gold are employed to explore the influence of hydrogen-bonding interactions on gas-surface energy exchange and accommodation. H-bonding, $\mathrm{COOH}$-terminated SAMs are found to produce more impulsive scattering and less thermal accommodation than non-H-bonding, $\mathrm{COOCH}_{3}$-terminated monolayers. For carbamatefunctionalized SAMs of the form $\mathrm{Au} / \mathrm{S}\left(\mathrm{CH}_{2}\right)_{16} \mathrm{OCONH}\left(\mathrm{CH}_{2}\right)_{n-1} \mathrm{CH}_{3}$, impulsive scattering decreases and accommodation increases as the H-bonding group is positioned farther below the terminal $\mathrm{CH}_{3}$.
\end{abstract}

PACS numbers: $34.50 . \mathrm{Lf}$

Chemical reactions between gas and solid phase molecules are mediated by an initial collision that determines whether the gas scatters directly away from the surface or accommodates with the material. Although the dynamics of gas-surface collisions have been explored extensively for atoms and molecules impinging on a variety of inorganic materials, the often-complex nature of organic surfaces has limited the number of detailed investigations into gas-surface collisions on organic films. A complete understanding of gas-surface chemistry on organic materials should address how the various impact sites and surface characteristics, such as interfacial hydrogen bonding, influence the outcome of the initial collision. The primary objective of the experimental studies presented below is to begin to address these issues by employing selfassembled monolayers (SAMs) of functionalized alkanethiols on gold and atomic-beam scattering techniques to explore how the molecular structure and the hydrogenbonding nature of an organic surface influence the extent of energy transfer and accommodation in gas-surface collisions.

Self-assembled monolayers of alkanethiols on gold provide model systems for fundamental studies into a variety of chemical and physical phenomena. In particular, the ability to place hydrogen-bonding groups at the terminus, as well as at specific locations along the alkanethiol chains, has prompted recent research into how intramonolayer forces affect structure [1], monolayer phase segregation [2], monolayer stability [3], gas-surface adsorption [4], and the dynamics of gas-surface energy exchange $[5,6]$. The studies discussed here are designed to extend the previous work on the energy exchange dynamics of gas-surface collisions by scattering argon from a series of carbonyl-containing SAMs.

A schematic of the SAM terminal groups used in these studies is shown in Fig. 1. The acid, $\mathrm{COOH}$, and ester, $\mathrm{COOCH}_{3}$, terminated SAMs are created by spontaneous assembly from $1 \mathrm{mM}$ ethanolic solutions of the corresponding $\omega$-functionalized alkanethiols onto gold-coated glass or mica [7] (the scattering results were found to depend only slightly on the underlying substrate). The 16mercaptohexadecanoic acid was purchased from Aldrich and used without further purification. The methyl-11mercaptoundecanoate was synthesized according to two previously established routes; see Refs. [8,9]. The two routes produced similar reflection absorption infrared spectroscopy (RAIRS) results and nearly identical Ar time-of-flight (TOF) scattering data. The carbamatefunctionalized SAMs [Fig. 1(b)] were synthesized via the exposure of 16-carbon OH-terminated SAMs to isocyanates of varying chain length: $\mathrm{OCN}\left(\mathrm{CH}_{2}\right)_{n-1} \mathrm{CH}_{3}$. Carbamate SAMs created via this vapor-phase exposure technique have been described in detail elsewhere [10]. In general, the monolayers are well ordered, with hydrogen-bonding interactions between the $\mathrm{C}=\mathrm{O}$ and $\mathrm{HN}$ groups of adjacent chains located $n$ carbon atoms below the surface [10]. Throughout the following discussion, we refer to structures (a), (b), and (c) in Fig. 1 as $\mathrm{COOH}-T, \mathrm{CONH}\left(\mathrm{CH}_{2}\right)_{n-1} \mathrm{CH}_{3}-T$, and $\mathrm{COOCH}_{3}-T$ SAMs, respectively.

RAIRS was performed using a Bruker IFS 66v/S spectrometer equipped with optics customized for grazing incidence reflection. Spectra were collected using $p$-polarized light at an incident angle of $86^{\circ}$ and a liquid $\mathrm{N}_{2}$ cooled MCT detector. Clean gold substrates were used as background references.
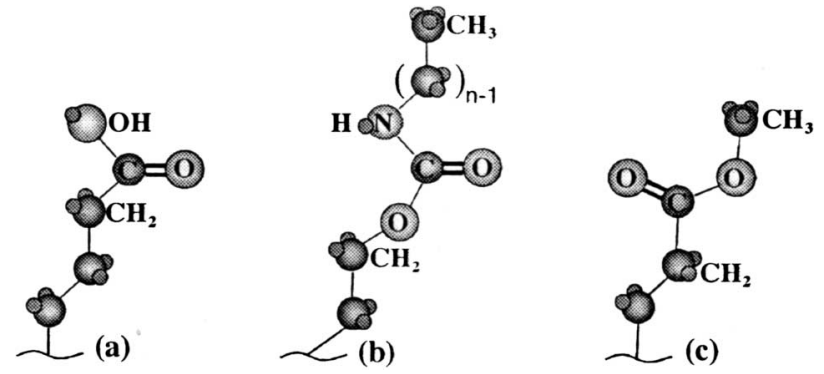

FIG. 1. Schematic model of the acid (a), carbamate (b), and ester (c) terminal groups. 
The scattering experiments were performed by directing a nearly monoenergetic Ar atomic beam at a particular SAM [5]. The peak incident energy was $E_{i}=$ $80 \mathrm{~kJ} / \mathrm{mol}$ and the incident angle was set to $30^{\circ}$ with respect to the surface normal. The flight times of the Ar atoms were recorded as they traveled from a spinning slotted wheel, scattered from the monolayer, and passed through an aperture into a mass spectrometer located at the specular angle of $30^{\circ}$. The raw spectrometer signal in the TOF distributions is proportional to the number density $N(t)$ and is used to compute the probability, $P\left(E_{f}\right)$, that an $\operatorname{Ar}$ atom leaves the surface with final energy, $E_{f}$.

Figures 2(a) and 2(b) show the time-of-flight and the corresponding $P\left(E_{f}\right)$ distributions for Ar atoms scattered from SAMs terminated by three different functional groups: $\mathrm{COOH}, \mathrm{CONHCH}_{3}$, and $\mathrm{COOCH}_{3}$. The spectra are composed of varying contributions of two scattering channels: a high-energy direct impulsive component (IC) and a slow, low-energy channel that appears to fit a Boltzmann distribution at the temperature of the surface [Boltzmann component (BC)]. The surface samples were
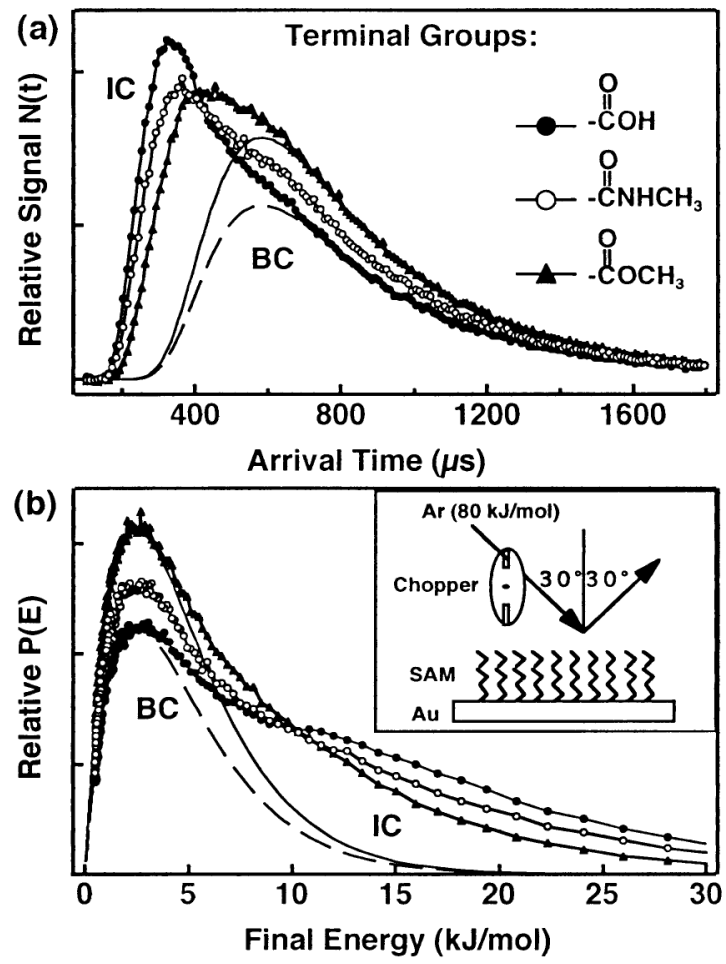

FIG. 2. (a) TOF distributions for $80 \mathrm{~kJ} / \mathrm{mol}$ Ar scattering from the acid-, methyl carbamate-, and methyl esterterminated SAMs. (b) The corresponding $P\left(E_{f}\right)$ distributions for data in (a). The solid and dashed curves represent BC at the temperature of the surface, scaled to fit the experimental data. The dashed curve is fit to the $\mathrm{COOH}-T$ scattering data and the solid curve is fit to the $\mathrm{COOCH}_{3}-T$ data. The IC label highlights the impulsive component of the scattered TOF and $P\left(E_{f}\right)$ distributions. The inset is a schematic representation of the experimental arrangement. held at room temperature for the data presented here. The energy distributions are separated into the impulsive and Boltzmann components by assigning the $\mathrm{BC}$ channel to the component of $P\left(E_{f}\right)$ that falls within a Boltzmann distribution: $\quad P_{\mathrm{BC}}\left(E_{f}\right)=E_{f}\left(R T_{\text {surf }}\right)^{-2} \exp \left(-E_{f} / R T_{\text {surf }}\right)$. The impulsive component is assigned to the difference between $P\left(E_{f}\right)$ and $P_{\mathrm{BC}}\left(E_{f}\right)$. The dynamics of gas-surface collisions are revealed through analysis of the fractional energy transferred to the surface for the impulsively scattered atoms, $\left(E_{i}-\left\langle E_{\mathrm{IC}}\right\rangle\right) / E_{i}$, and the fraction of atoms that recoil (at $\theta_{f}=30^{\circ}$ ) with a thermal distribution of velocities (BC fraction) [11]. While the fractional energy transfer for the impulsive component scales with the kinetic energy of the incident beam [5], the overall energy distribution is broadened by the virtually infinite number of different impact sites on the surface, such as above individual methyl groups, between two adjacent methyl groups, and the hollow sites between three terminal methyl groups.

Figure 2 provides a comparison of the TOF and corresponding $P\left(E_{f}\right)$ distributions for $80 \mathrm{~kJ} / \mathrm{mol}$ Ar scattering from SAMs terminated by $\mathrm{H}$-bonding and non- $\mathrm{H}-$ bonding groups. In accord with previous results, the overall energy transfer is extensive, $>75 \%$, for every monolayer studied [5]. This general trend is attributed to the numerous energetic degrees of freedom of the organic films including concerted waving motions of the chains, vibrational modes along the chains, and hindered rotations of the terminal groups. However, within this set of three SAMs, the scattering dynamics are found to depend on the particular functional groups at the termini of the monolayer chains. In particular, for the specular scattering conditions used in these experiments, direct impulsive scattering is the primary channel for atoms recoiling from the $\mathrm{COOH}-T$ surface while the $\mathrm{BC}$ dominates scattering from the $\mathrm{COOCH}_{3}-T$ monolayer. For the $\mathrm{CONHCH}_{3}-T$ SAM, the IC and $\mathrm{BC}$ contribute nearly equally to scattered $P\left(E_{f}\right)$ distributions. These differences in the scattering channels may be due to one or a combination of four key aspects that determine the rate of gas-surface energy transfer and thermalization: (i) overall monolayer structure and roughness, (ii) terminal group energetic degrees of freedom, (iii) gas-surface interaction potentials, and (iv) intramonolayer hydrogen bonding. Since all three of the monolayers represented in Fig. 2 contain a carbonyl group and an additional oxygen or nitrogen atom at the terminus of the monolayer, we speculate that the Ar-monolayer potential energy surfaces are similar. Therefore, the following discussion focuses on the influence of structure and hydrogen bonding in the scattering dynamics.

Figure 3 shows RAIR spectra for each class of SAMs used in the scattering experiments. Mode assignments for the primary transitions can be found in Refs. $[8,10]$. An important feature of each spectrum is the position of the $d^{-}$and $d^{+} \mathrm{CH}_{2}$ C-H stretching modes at 2918 and $2851 \mathrm{~cm}^{-1}$, respectively. These peak positions are the 


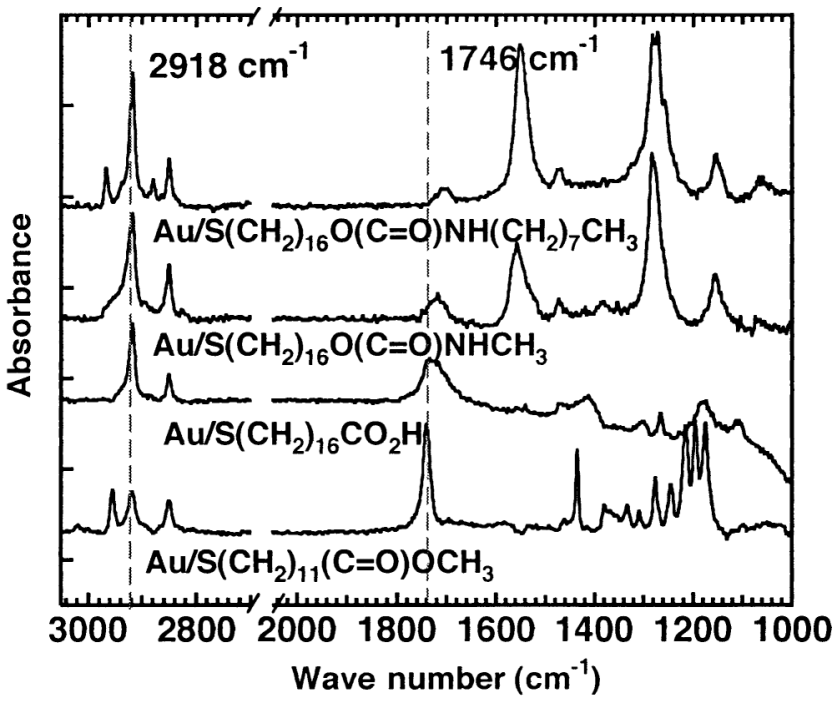

FIG. 3. RAIR spectra of SAMs formed from acidand methyl ester-terminated alkanethiols and carbamatefunctionalized SAMs formed via the vapor reaction technique described in Ref. [10].

same for each of the monolayers and are indicative of alltrans crystalline-like packing of the alkyl chains [7]. The evidence for forming well-ordered high-density monolayers is consistent with previous RAIRS work that demonstrates that the COOH-T and $\mathrm{COOCH}_{3}-T$ SAMs form similar highly organized structures [8]. The identical positions of the $\mathrm{C}-\mathrm{H}$ stretching frequencies for each SAM suggest that differences in the Ar scattering dynamics are not likely due to large variances in the packing density or structure of the monolayers. Furthermore, previous results indicate that the Ar TOF distributions are largely insensitive to relatively small differences in monolayer order or even surface roughness [5].

An alternative explanation for the observed trends in the scattering dynamics focuses on the obvious differences in the available energetic degrees of freedom of the three terminal groups. We speculate that the additional degrees of freedom of the $\mathrm{COOCH}_{3}-T \mathrm{SAM}$, as compared to the COOH-T SAM, are partly responsible for the observed differences in the scattering dynamics. The outer methyl group of the ester offers additional bending, stretching, and torsional modes that may facilitate energy exchange. However, it is unlikely that the additional degrees of freedom of the methyl group alone account for the trends in scattering. Classical trajectory calculations indicate that the energy modes of terminal methyl groups in $n$-alkanethiol SAMs play a minor role in the energy transfer dynamics involving high-energy rare-gas collisions [12]. In addition, previous Ar scattering studies have revealed that for $80 \mathrm{~kJ} / \mathrm{mol}$ Ar scattering from $\mathrm{CH}_{3}-T$ SAMs, as many as six carbon atoms along the alkane chains participate in dissipating the collision energy [6]. These results suggest that the terminal groups are not as important as the ability to excite motions that involve many atoms along the alkane chains.
Previous Ar scattering studies comparing $\mathrm{COOH}-$, $\mathrm{NH}_{2^{-}}, \mathrm{OH}-$, and $\mathrm{CH}_{3}$-terminated SAMs show that the hydrogen-bonding monolayers produce much more impulsive scattering than the non-hydrogen-bonding system [5]. The data from the previous work imply that hydrogen-bonding interactions increase the rigidity of a surface relative to an analogous non-hydrogen-bonding system [5]. Hydrogen bonds may anchor the terminal groups thereby rendering a monolayer less flexible than a methyl-terminated monolayer. This hypothesis is consistent with the TOF and $P\left(E_{f}\right)$ data presented in Fig. 2, which show that the hydrogen-bonding surfaces, $\mathrm{COOH}-T$ and $\mathrm{CONHCH}_{3}-T$ SAMs, produce more impulsive scattering and less accommodation than the nonhydrogen-bonding $\mathrm{COOCH}_{3}-T$ SAM.

Spectroscopic evidence for the extent of hydrogen bonding within these monolayers is provided by the RAIR data of Fig. 3. The $\mathrm{COOH}$ and $\mathrm{CONHCH}_{3}$ SAMs exhibit broad redshifted carbonyl peaks at $\sim 1700 \mathrm{~cm}^{-1}$. In addition, this peak is relatively weak, suggesting that the $\mathrm{C}=\mathrm{O}$ bond lies predominantly parallel to the plane of the gold surface, which should facilitate hydrogen bonding with adjacent hydrogen-bond donor groups, $\mathrm{OH}$ and $\mathrm{NH}$ [8]. In contrast, the strong narrow peak at $1746 \mathrm{~cm}^{-1}$ in the RAIR spectrum of the $\mathrm{COOCH}_{3}-T$ SAM is indicative of a free non-hydrogen-bonding carbonyl. The correlation of relatively large impulsive scattering components with the hydrogen-bonding character of the monolayers suggests that hydrogen-bonding interactions are responsible for the rigid nature of these monolayers. It appears that hydrogen bonding may dampen low-energy modes within the monolayer to make the individual alkane chains less flexible than the free, non-hydrogen-bonding monolayer.

The above discussions suggest that hydrogen-bonding groups near the terminus of a SAM create a more rigid collision partner than an analogous non-hydrogenbonding system. This section explores the ability of hydrogen-bonding groups buried farther below the methyl terminus to influence the outcome of the gassurface collision. In addition to the $\mathrm{CONHCH}_{3}-T$ monolayer, Fig. 3 shows a RAIR spectrum of a $\mathrm{CONH}\left(\mathrm{CH}_{2}\right)_{7} \mathrm{CH}_{3}-T$ SAM. The two spectra are very similar with sharp C-H stretching peaks at 2918 and $2851 \mathrm{~cm}^{-1}$, a broad and weak carbonyl peak at about $1700 \mathrm{~cm}^{-1}$, and the expected low-energy carbamate stretches [10]. These characteristics are similar for the entire range of $\mathrm{CONH}\left(\mathrm{CH}_{2}\right)_{n-1} \mathrm{CH}_{3}-T$ SAMs studied [10]. The primary difference within the RAIR spectra is in the intensity of the methylene $\mathrm{C}-\mathrm{H}$ modes, which scale with the chain length, $n$. In general, the RAIR data indicate that the monolayers form well-ordered and highly dense structures with significant $\mathrm{C}=\mathrm{O}-\mathrm{-HN}$ hydrogen-bonding interactions located $n$ methylene groups below the outermost methyl group [10].

Figure 4 shows the relative TOF and $P\left(E_{f}\right)$ distributions for $80 \mathrm{~kJ} / \mathrm{mol} \mathrm{Ar}$ scattering from the 

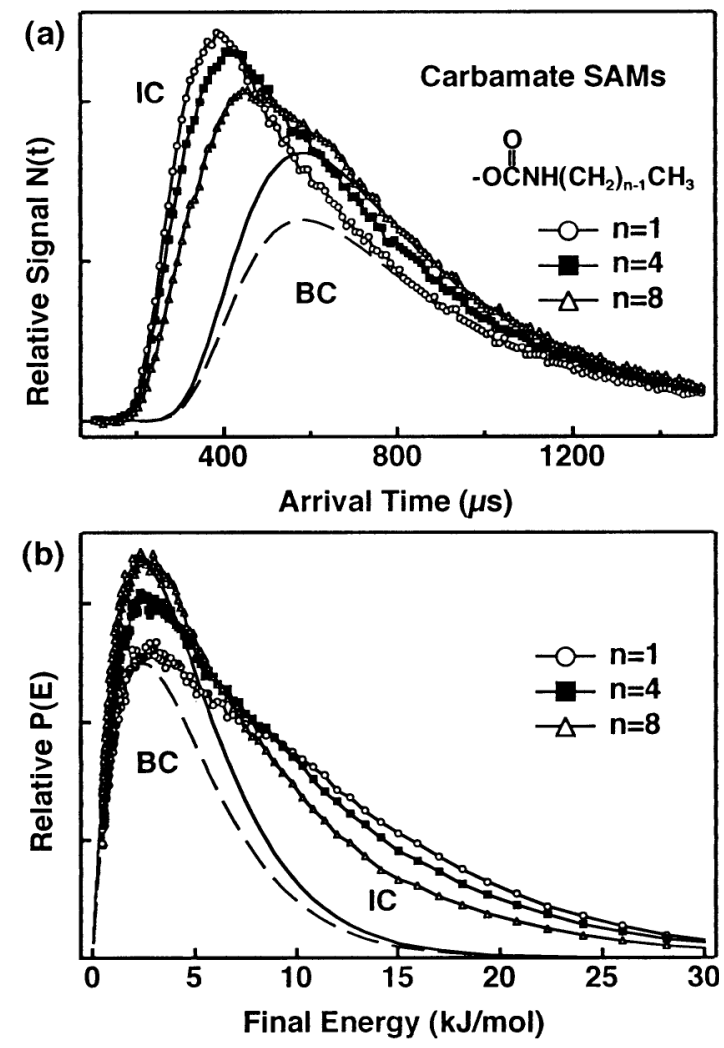

FIG. 4. (a) TOF distributions for $80 \mathrm{~kJ} / \mathrm{mol}$ Ar scattering from the carbamate-terminated SAMs with $n$ number of total carbon atoms above the hydrogen-bonding group. (b) The corresponding $P\left(E_{f}\right)$ distributions for data in (a). The solid and dashed curves represent $\mathrm{BC}$ at the temperature of the surface, scaled to fit the experimental data. The dashed curve is fit to the $n=1$ data and the solid curve is fit to the $n=8$ data. The IC label highlights the impulsive component of the scattered TOF and $P\left(E_{f}\right)$ distributions.

$\mathrm{CONH}\left(\mathrm{CH}_{2}\right)_{n-1} \mathrm{CH}_{3}-T$ SAMs with $n=1,4$, and 8. The data indicate that the Boltzmann component increases in intensity with increasing $n$ while, concomitantly, the impulsive component decreases in intensity and shifts to lower final energies (later arrival times). The Boltzmann fractions for the entire range of monolayers studied are plotted as a function of chain length in Fig. 5. The data indicate that the fraction of atoms that leave the surface in a Boltzmann distribution increases from $n=1$ to $n=5$ and becomes constant for $n>5$. Since all of the carbamate SAMs are expected to have similar packing densities, order, and orientation [10], we hypothesize that the observed trend in the energy exchange dynamics is due to the increasing degrees of freedom above the hydrogen-bonding group as $n$ increases.

The effect of alkane chain length in rare-gas scattering from SAMs has been explored in detail in previous studies [6]. The investigations revealed that, for collisions of $80 \mathrm{~kJ} / \mathrm{mol} \mathrm{Ar}$ on $n$-alkanethiol SAMs, long-range molecular motions involving up to six methylene groups along the individual chains participate in the energy

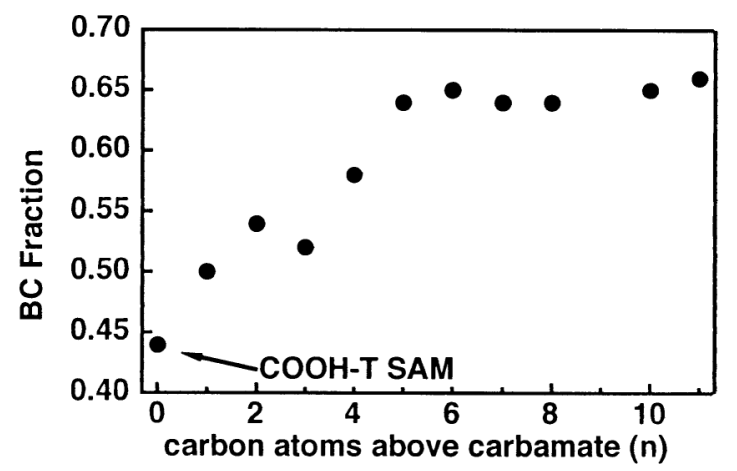

FIG. 5. BC fraction as a function of the number of carbon atoms above the carbamate functional group.

transfer dynamics. This observation agrees well with the expected time scale for the impulsive gas-surface collision and the rate at which energy propagates along the chains [6]. Therefore, we suggest that the trend reported in Fig. 5 can be attributed to the same effect. Within this picture, the $\mathrm{C}=\mathrm{O}-\mathrm{-HN}$ hydrogen-bonding network serves as a rigid platform and the non-hydrogen-bonding alkane chains above the network provide an effective energy sink for dissipating the translational energy of the impinging Ar atoms. As in scattering from the $n$-alkanethiol SAMs on gold, we find that 5-6 methylene groups are needed to fully shield the underlying rigid platform.

[1] G. E. Poirier, E. D. Pylant, and J. M. White, J. Chem. Phys. 104, 7325 (1996); J. P. Folkers, P. E. Laibinis, and G. M. Whitesides, Langmuir 8, 1330 (1992).

[2] R. K. Smith, S. M. Reed, P. A. Lewis, J. D. Monnell, R. S. Clegg, K. F. Kelly, L. A. Bumm, J. E. Hutchison, and P. S. Weiss, J. Phys. Chem. B 105, 1119 (2001).

[3] R. Valiokas, M. Ostblom, S. Svedhem, S. C. T. Svensson, and B. Liedberg, J. Phys. Chem. B 106, 10401 (2002).

[4] L. Bertilsson, K. Potje-Kamloth, H. D. Liess, and B. Liedberg, Langmuir 15, 1128 (1999); A. D. Vogt and T. P. Beebe, J. Phys. Chem. B 103, 8482 (1999).

[5] B.S. Day, S. F. Shuler, A. Ducre, and J. R. Morris, J. Chem. Phys. 119, 8084 (2003).

[6] B. S. Day and J. R. Morris, J. Phys. Chem. B 107, 7120 (2003).

[7] R. G. Nuzzo, L. H. Dubois, and D. L. Allara, J. Am. Chem. Soc. 112, 558 (1990).

[8] I. Engquist, M. Lestelius, and B. Liedberg, Langmuir 13, 4003 (1997).

[9] C. D. Bain, E. B. Troughton, Y. Tao, J. Evall, G. M. Whitesides, and R. G. Nuzzo, J. Am. Chem. Soc. 111, 321 (1989).

[10] M. K. Ferguson, E. R. Low, and J. R. Morris, Langmuir (to be published).

[11] D. R. Miller, Atomic and Molecular Beam Methods (Oxford University Press, New York, 1988).

[12] T. Yan and W. L. Hase, J. Phys. Chem. B 106, 8029 (2002). 